\title{
On Some Indefinite Integrals of Confluent Hypergeometric Functions*
}

\author{
Edward W. Ng** and Murray Geller**
}

(January 5, 1970)

\begin{abstract}
Analytical expressions and reduction formulas are developed for various indefinite integrals of the confluent hypergeometric functions. These integrals are of the type $\int f(a, b, z) z^{p} e^{\alpha z} d z$, where $f$ is one of the two Kummer functions $M(a, b, z) \equiv{ }_{1} F_{1}(a ; b ; z)$ or $U(a, b, z)$, with real or complex $a, b, z$ and $\alpha$, and real $p$.
\end{abstract}

Keys words: Bessel functions; confluent hypergeometric functions; electronic energies; indefinite integrals.

\section{Introduction}

Multiple integrals occur in many physical problems in the form where one of the integrations results in a higher mathematical function. Thus one is often faced with the task of evaluating integrals involving higher functions. Whereas there exists a large body of literature for integrals with limits $(0, \infty)$ and $(-\infty, \infty)$, there are considerably fewer sources pertaining to indefinite integrals. This latter type is important, for example, in statistical mechanics for the integration of a distribution over a finite portion of velocity or momentum space and in electromagnetic theory for the integration over a finite charge configuration. We note the appearance of only one major monograph with emphasis on indefinite and finite integrals of higher functions, that of Y. L. Luke $[1]^{1}$ on Integrals of Bessel Functions. This monograph covers a much wider range of material than its title suggests.

In this note we investigate some indefinite integrals involving confluent hypergeometric functions of the type $\int f(a, b, z) z^{p} e^{\alpha z} d z$, where $f$ is one of the two Kummer functions $M(a, b, z) \equiv{ }_{1} F_{1}(a$; $b ; z)$ or $U(a, b, z)$. Several recent applications of these integrals to studies on molecular electronic energies $[2,3]$ have been made.

It is well known that the confluent hypergeometric functions contain many other higher functions as special cases. For the definitions and an extensive analysis of the properties of these functions, the reader is referred to the Handbook of Mathematical Functions [4] and the work by Slater [5].

We define the integrals to be considered in this note as follows: ${ }^{2}$

and the special cases

$$
\begin{aligned}
& M_{p}(\alpha ; a, b, z) \equiv \int M(a, b, z) z^{p} e^{\alpha z} d z, \\
& U_{p}(\alpha ; a, b, z) \equiv \int U(a, b, z) z^{p} e^{\alpha z} d z,
\end{aligned}
$$

$$
\begin{aligned}
& \Phi_{n}(a, b, z) \equiv M_{n}(0 ; a, b, z), \\
& \Psi_{n}(a, b, z) \equiv U_{n}(0 ; a, b, z) .
\end{aligned}
$$

*An invited paper. This paper presents the results of one phase of research carried out at the Jet Propulsion Laboratory, California Institute of Technology, under Contract NAS7-100, sponsored by the National Aeronautics and Space Administration. Acknowledgment is made to Dr. M. Saffren for helpful discussions.

**Present address: Jet Propulsion Laboratory, California Institute of Technology, Pasadena, Calif. 91103.

' Figures in brackets indicate the literature references at the end of this paper.

2 Throughout this paper we omit the constant for all indefinite integrals. 
Here $a, b, z$, and $\alpha$ may be real or complex, $p$ is real and $n$ is a positive integer or 0 . In section 2 we discuss the function $\Phi_{n}$ and in section 3 the function $\Psi_{n}$ showing how both of these functions can be reduced to simple linear combinations of elementary functions and various hypergeometric functions. In section 4 we consider eq (1) with $\alpha=0$, and we conclude in section 5 with a brief discussion of the general integrals $M_{p}(\alpha ; a, b, z)$ and $U_{p}(\alpha ; a, b, z)$ of eq (1), relating them to some recent work by Babister [6]. In an appendix we specialize the parameters in $M_{p}(\alpha ; a, b, z)$ and $U_{p}(\alpha ; a, b, z)$ to the Bessel function case, $b=2 a$, and, using the results of sections 4 and 5 , display several interesting indefinite integrals for the Bessel functions.

\section{Analysis of $\Phi_{n}(a, b, z)$}

In this section we analyze the function $\Phi_{n}(a, b, z)$ and show its reduction to elementary functions or to linear combinations of various hypergeometric functions. We use the following well known properties of the $M(a, b, z)[4,5]$

$$
\begin{gathered}
(1+a-b) M(a, b, z)-a M(a+1, b, z)+(b-1) M(a, b-1, z)=0, \\
b M(a, b, z)-b M(a-1, b, z)-z M(a, b+1, z)=0, \\
\frac{d}{d z} M(a, b, z)=\frac{a}{b} M(a+1, b+1, z) ;
\end{gathered}
$$

and their equivalent integrated expressions (see footnote 2)

$$
\begin{gathered}
(1+a-b) \Phi_{n}(a, b, z)-a \Phi_{n}(a+1, b, z)+(b-1) \Phi_{n}(a, b-1, z)=0, \\
b \Phi_{n}(a, b, z)-b \Phi_{n}(a-1, b, z)-\Phi_{n+1}(a, b+1, z)=0, \\
z^{n} M(a, b, z)-n \Phi_{n-1}(a, b, z)=\frac{a}{b} \Phi_{n}(a+1, b+1, z), \\
M(a, b, z)=\frac{a}{b} \Phi_{0}(a+1, b+1, z) .
\end{gathered}
$$

Equations (5a) and (5b) are obtained by integrating eq (5) by parts. We also note the useful expressions

$$
\begin{gathered}
M(0, b, z)=1, \\
\Phi_{n}(0, b, z)=\frac{z^{n+1}}{n+1}
\end{gathered}
$$

Four different cases arise dependent on whether $a$ is equal or unequal to an integer and $b$ is equal or unequal to an integer.

\section{(i) $a \neq$ integer, $b \neq$ integer}

Use of eq (5a) with $a$ replaced by $a-1$ and $b$ replaced by $b-1$ yields the reduction formula

$$
\Phi_{n}(a, b, z)=\frac{(b-1)}{(a-1)} z^{n} M(a-1, b-1, z)-n \frac{(b-1)}{(a-1)} \Phi_{n-1}(a-1, b-1, z) .
$$

Repeated application of eq (7) generates $\Phi_{n}(a, b, z)$ as a linear combination of terms 
$z^{n-n^{\prime}} M\left(a-n^{\prime}-1, b-n^{\prime}-1, z\right)$, with $n^{\prime}$ ranging from 0 to $n-1$, plus the lowest order term, $\Phi_{0}(a-n, b-n, z)$, which is given by eq $(5 \mathrm{~b})$.

(ii) $a \neq$ integer, $b=$ integer $>0$

We first note that the integral, as well as $M(a, b, z)$, is undefined for $b=-m$. For $b \neq 1$ we can replace $(b-1) \Phi_{n-1}(a-1, b-1, z)$ in eq (7) by its equivalent from eq (3a) with $a$ replaced by $a-1$ and $n$ replaced by $n-1$, to vield a reduction formula analogous to eq (7)

$$
\Phi_{n}(a, b, z)=\frac{(b-1)}{(a-1)} z^{n} M(a-1, b-1, z)-n \Phi_{n-1}(a, b, z)+n \frac{(a-b)}{(a-1)} \Phi_{n-1}(a-1, b, z) .
$$

Repeated application of eq (8) generates for $\Phi_{n}$ a linear combination of $M$ 's plus the lowest order terms $\Phi_{0}\left(a^{\prime}, b^{\prime}, z\right)$ which we obtain from eq $(5 b)$. For the case $b=1$ we see that eq (8) is still valid if we recognize from eqs (3) and (4) that

$$
\operatorname{Lim}_{b \rightarrow 1}(b-1) M(a-1, b-1, z)=(a-1) z M(a, 2, z) .
$$

Substituting eq (9) into eq (8), we have the reduction formula

$$
\Phi_{n}(a, 1, z)=z^{n+1} M(a, 2, z)-n\left[\Phi_{n-1}(a, 1, z)-\Phi_{n-1}(a-1,1, z)\right],
$$

with the lowest order term being given by

$$
\Phi_{0}(a, 1, z)=z M(a, 2, z) .
$$

One can easily show by mathematical induction that the general result for the case $b=1$ is

$$
\Phi_{n}(a, 1, z)=z^{n+1} \sum_{m=0}^{n}(-1)^{m}\left(\begin{array}{c}
n \\
m
\end{array}\right) \frac{1}{(m+1)} M(a, m+2, z)
$$

which holds equally well when $a$ is an integer.

$$
\text { (iii) } a=\text { integer, } b \neq \text { integer }
$$

For $a>n+1$, eq (7) can be used to reduce $\Phi_{n}(a, b, z)$ to a series of $M$ 's plus the lowest order term $\Phi_{0}(a-n, b-n, z)$ given by eq (5b). For $a=n+1$ and $a=n>0$, repeated application of eq (7) again reduces $\Phi_{n}$ to a series of $M$ 's plus the lowest order terms $\Phi_{0}(1, b-n, z)$ and $\Phi_{1}(1, b-n+1, z)$ respectively. These are related through eq (4a) with $a=1$ and $n=0$ and eq (6a) by

$$
\Phi_{1}(1, b-n+1, z)=(b-n)\left[\Phi_{0}(1, b-n, z)-z\right] .
$$

The primitive function $\Phi_{0}\left(1, b^{\prime}, z\right)$, not reducible any further, can be written [1, p. 5]

$$
\Phi_{0}\left(1, b^{\prime}, z\right)=z_{2} F_{2}\left(1,1 ; 2, b^{\prime} ; z\right) .
$$

For the special case where $b^{\prime}$ is an integer, the ${ }_{2} F_{2}$ function can be reduced to incomplete gamma functions and exponential integrals as will be seen in case (iv). The condition $n>a>0$ is easily handled by repeated use of eq (4a) with $n$ replaced by $n-1$ and $b$ replaced by $b-1$

$$
\Phi_{n}(a, b, z)=(b-1)\left[\Phi_{n-1}(a, b-1, z)-\Phi_{n-1}(a-1, b-1, z)\right],
$$

until the reduction generates either of the lowest order terms $\Phi_{n^{\prime}}(0, b, z)$ given by eq $(6 a), \Phi_{0}\left(a^{\prime} \neq 1\right.$, $\left.b^{\prime}, z\right)$ of eq $(5 \mathrm{~b})$, or $\Phi_{0}\left(1, b^{\prime}, z\right)$, the primitive given in eq (14). Lastly, for the case $a=-m, m=0,1$, $2, \ldots$. since $M(-m, b, z)$ is a polynomial of $m+1$ terms we can either employ eq (5a) backwards until we arrive at $\Phi_{n+m}(0, b+m, z)$, given by eq $(6 a)$, or directly integrate to obtain 


$$
\Phi_{n}(-m, b, z)=\sum_{k=0}^{m}(-1)^{k}\left(\begin{array}{c}
m \\
k
\end{array}\right) \frac{z^{n+k+1}}{(b)_{k}(n+k+1)},
$$

where

$$
\begin{gathered}
(b)_{k}=\Gamma(b+k) / \Gamma(b) . \\
\text { (iv) } a=\text { integer, } b=\text { integer }>0 .
\end{gathered}
$$

With $a \geqslant b$, repeated use of eq (4) generates

$$
M(b+m, b, z)=e^{z} \sum_{k=0}^{m}\left(\begin{array}{c}
m \\
k
\end{array}\right) \frac{z^{k}}{(b)_{k}},
$$

since

$$
M(b, b, z)=e^{z},
$$

which holds even when $b \neq$ integer. Integration of eq (17) obviously yields

$$
\Phi_{n}(b+m, b, z)=\sum_{k=0}^{m}\left(\begin{array}{c}
m \\
k
\end{array}\right) \frac{1}{(b)_{k}} \int z^{n+k} e^{z} d z .
$$

For the case $b>a>0$, we have from eq (3a) with $b=a+m$, the recursion relation

$$
(1-m) \Phi_{n}(a, a+m, z)=a \Phi_{n}(a+1, a+m, z)-(a+m-1) \Phi_{n}(a, a+m-1, z) .
$$

Equation (20) is repeatedly applied until the right hand side contains only terms of the form $b=a+1$. Using eq (4a) with $b$ replaced by $a$ and $n$ replaced by $n-1$, we have the reduction formula

$$
\Phi_{n}(a, a+1, z)=a \int z^{n-1} e^{z} d z-a \Phi_{n-1}(a-1, a, z) .
$$

The reduction by eq (2l) is continued, generating a series of terms $\int z^{k} e^{z} d z$ plus the lowest order term. If $a \leqslant n$, the lowest order term is $\Phi_{n-a}(0,1, z)$, which is given by eq $(6 \mathrm{a})$. With $a=n+1$, the lowest order term is

$$
\Phi_{0}(1,2, z)=\int\left(e^{z}-1\right) \frac{d z}{z}
$$

or equivalently

$$
\begin{aligned}
& \Phi_{0}(1,2, x)=E i(x)-\ln x \quad x>0, \\
& \Phi_{0}(1,2,-z)=-E_{1}(z)-\ln z \quad|\arg z|<\pi,
\end{aligned}
$$

where $E_{1}$ and $E i$ are the exponential integrals [4, Chap. 5]. Finally, if $a>n+1$, the lowest order term is $\Phi_{0}(a-n, a-n+1, z)$. Using eq (5b), we can express this in terms of $M(a-n-1, a-n, z)$. Since

$$
M(n, n+1,-x)=\frac{n !}{x^{n}}\left[1-e^{-x} e_{n-1}(x)\right]
$$

where $e_{n}(x)=\sum_{k=0}^{n} \frac{x^{k}}{k !} ;$ we have

$$
\Phi_{0}(a-n, a-n+1, z)=(-1)^{a-n-1} \frac{(a-n)(a-n-2) !}{z^{a-n-1}}\left[1-e^{z} e_{a-n-2}(-z)\right] .
$$


We note that since

$$
\gamma(a, x)=a^{-1} x^{\prime \prime} e^{-x} M(1,1+a, x)=a^{-1} x^{\prime \prime} M(a, 1+a,-x),
$$

where $\gamma(a, x)$ is the incomplete gamma function [7], the $\Phi_{0}\left(1, b^{\prime}, z\right)$ of eq (14) can alternatively be written as

$$
\Phi_{0}\left(1, b^{\prime}, z\right)=\left(b^{\prime}-1\right) \int \gamma\left(b^{\prime}-1, z\right) z^{1-b^{\prime}} e^{z} d z
$$

whereas $\Phi_{0}(a, 1+a,-x)$ can be expressed as

For $a \neq 1$, this reduces to

$$
\Phi_{0}(a, 1+a,-x)=a \int \gamma(a, x) x^{-a} d x,
$$

$$
\Phi_{0}(a, a+1,-x)=\frac{a}{1-a}\left[x^{1-a} \gamma(a, x)+e^{-x}\right] .
$$

For $a=1$, eq. (28a) reduces to eq. (23b), and with $a=$ integer $>1$, eq (28b) reduces to eq (25).

The last case to be considered in this section is $b>a$ where $b>0$ and $a=-m$. This case is immediately obtainable from eq. (16) since that equation obviously holds for $b=$ integer $>0$.

\section{Analysis of $\Psi_{n}(a, b, z)$}

Analogous to the results of section 2, the function $\Psi_{n}(a, b, z)$ can be reduced to elementary functions or to linear combinations of various hypergeometric functions. Here, too, four different cases, $a$ equal or unequal to an integer and $b$ equal or unequal to an integer, must be considered for the complete reduction of $\Psi_{n}$. We commence the analysis with the definition of $U(a, b, z)[5]$

$$
U(a, b, z)=\frac{\pi}{\sin \pi b}\left[\frac{M(a, b, z)}{\Gamma(1+a-b) \Gamma(b)}-z^{1-b} \frac{M(1+a-b, 2-b, z)}{\Gamma(a) \Gamma(2-b)}\right],
$$

and the well known properties $[4,5]$

$$
\begin{gathered}
U(a, b, z)-a U(a+1, b, z)-U(a, b-1, z)=0, \\
(b-a) U(a, b, z)+U(a-1, b, z)-z U(a, b+1, z)=0, \\
\frac{d}{d z} U(a, b, z)=-a U(a+1, b+1, z) .
\end{gathered}
$$

We also note the integrated form of eqs $(30)-(32)^{2}$

$$
\begin{gathered}
\Psi_{n}(a, b, z)-a \Psi_{n}(a+1, b, z)-\Psi_{n}(a, b-1, z)=0, \\
(b-a) \Psi_{n}(a, b, z)+\Psi_{n}(a-1, b, z)-\Psi_{n+1}(a, b+1, z)=0, \\
z^{n} U(a, b, z)-n \Psi_{n-1}(a, b, z)=-a \Psi_{n}(a+1, b+1, z), \\
U(a, b, z)=-a \Psi_{0}(a+1, b+1, z) .
\end{gathered}
$$

Equation (32b) is simply obtained from eq (32a) by setting $n=0$. Other properties of the $U(a, b, z)$ used in this analysis are

$$
\begin{gathered}
U(0, b, z)=1, \\
\Psi_{n}(0, b, z)=z^{n+1} /(n+1), \\
U(a, a+1, z)=z^{-a}, \\
U(a, 1-n, z)=z^{n} U(a+n, 1+n, z),
\end{gathered}
$$


the last equation being a special case of Kummer's transformation. One final property of the $U(a, b, z)$, as opposed to the $M(a, b, z)$, is that they are defined for all values of $a$ and $b$.

\section{(i) $a \neq$ integer, $b \neq$ integer}

Using eq (32a) with $a$ replaced by $a-1$ and $b$ replaced by $b-1$, we have

$$
\Psi_{n}(a, b, z)=\frac{1}{(1-a)}\left[z^{n} U(a-1, b-1, z)-n \Psi_{n-1}(a-1, b-1, z)\right] .
$$

Repeated use of eq (36) reduces $\Psi_{n}$ to a series of terms $z^{n-n^{\prime}} U\left(a-n^{\prime}-1, b-n^{\prime}-1, z\right)$ plus the lowest order term, which by eq $(32 \mathrm{~b})$ is given by

$$
\Psi_{0}\left(a^{\prime}, b^{\prime}, z\right)=\frac{1}{\left(1-a^{\prime}\right)} U\left(a^{\prime}-1, b^{\prime}-1, z\right),
$$

or equivalently, from eq (30) with $a$ replaced by $a-1$

$$
\Psi_{0}\left(a^{\prime}, b^{\prime}, z\right)=\frac{1}{\left(1-a^{\prime}\right)} U\left(a^{\prime}-1, b^{\prime}, z\right)+U\left(a^{\prime}, b^{\prime}, z\right) .
$$

\section{(ii) $a \neq$ integer, $b=$ integer}

For $b \geqslant 1$, if we replace both terms on the right hand side of eq (36) by their equivalents from eq (30) and (30a) with $a$ replaced by $a-1$, then we have the reduction formula for $\Psi_{n}$

$$
\begin{aligned}
\Psi_{n}(a, b, z)=\left[z^{n}\{U(a-1, b, z)+(1-a) U\right. & (a, b, z)\} \\
& \left.-n\left\{\Psi_{n-1}(a-1, b, z)+(1-a) \Psi_{n-1}(a, b, z)\right\}\right] ;
\end{aligned}
$$

the lowest order term is given by eq (38). If $b \leqslant 0$, we use the integrated form of Kummer's transformation, eq (35), to yield

$$
\Psi_{n}(a,-m, z)=\Psi_{n+m+1}(a+m+1, m+2, z),
$$

which can obviously be handled by means of eqs (39) and (38) above.

\section{(iii) $a=$ integer, $b \neq$ integer}

If $a=-m, m=0,1,2, \ldots$, then from eq (31a) with $a=-m+1$, we have the recursion relation

$$
\Psi_{n}(-m, b, z)=\Psi_{n+1}(-m+1, b+1, z)-(b+m-1) \Psi_{n}(-m+1, b, z),
$$

which reduces to $\Psi_{n^{\prime}}\left(0, b^{\prime}, z\right)$ of eq (33a) upon repeated usage. An alternative approach is to note that since

$$
U(-m, b, z)=(-1)^{m} m ! L_{m}^{(b-1)}(z),
$$

where $L_{m}^{(\alpha)}(z)$ is the associated Laguerre function [4, chap. 22], direct integration yields

$$
\Psi_{n}(-m, b, z)=(-1)^{m} m ! \sum_{k=0}^{m}\left(\begin{array}{c}
m+b-1 \\
m-k
\end{array}\right) \frac{(-1)^{k}}{k !} \frac{z^{n+k+1}}{(n+k+1)} .
$$


For $a>0$, three distinct situations arise. If $a>n+1$, use of eq (36) reduces $\Psi_{n}(a, b, z)$ to a series of $U$ 's plus the lowest order term $\Psi_{0}(a-n, b-n, z)$ given by eq (37) as in case (i) above. With $a=n$ or $a=n+1$, repeated application of eq (36) reduces $\Psi_{n}$ to a series of $U$ 's plus the lowest order terms $\Psi_{0}(1, b-n, z)$ and $\Psi_{1}(1, b-n+1, z)$ respectively. These are related through eq (3la) with $n=0$ and $a=1$ by

$$
\Psi_{1}\left(1, b^{\prime}+1, z\right)=z+\left(b^{\prime}-1\right) \Psi_{0}\left(1, b^{\prime}, z\right) .
$$

Furthermore, from the definition of $U(a, b, z)$, eq (29), with $a=1$, we see that

$$
\Psi_{0}\left(1, b^{\prime}, z\right)=\Gamma\left(b^{\prime}-1\right) \int \frac{e^{z}}{z^{b^{\prime}-1}} d z-\frac{1}{\left(b^{\prime}-1\right)} \Phi_{0}\left(1, b^{\prime}, z\right)
$$

where the first term in eq (45) is an incomplete gamma function and the second term is precisely the primitive function discussed in section 2, eq (14). We remark that for the case $b=$ integer, eq (45) reduces to powers, logarithms and the exponential integral as will be seen in (iv) below. Finally, for $a<n$, use of eq (31a) with $n$ replaced by $n-1$ and $b$ replaced by $b-1$ leads to the reduction formula

$$
\Psi_{n}(a, b, z)=(b-a-1) \Psi_{n-1}(a, b-1, z)+\Psi_{n-1}(a-1, b-1, z) .
$$

Continued application of eq (46) results in the lowest order terms $\Psi_{n^{\prime}}\left(0, b^{\prime}, z\right)$ given by eq (33a) and $\Psi_{n^{\prime}}\left(n^{\prime}, b^{\prime}, z\right)$, the case $a=n$ discussed above.

$$
\text { (iv) } a=\text { integer, } b=\text { integer }
$$

Corresponding to eq (26) for the representation of the incomplete gamma function, $\gamma(a, x)$, in terms of $M$, we have

$$
\Gamma(a, x)=x^{a} e^{-x} U(1,1+a, x)=e^{-x} U(1-a, 1-a, x) .
$$

Therefore for the case $a=b=-m, m=0,1,2, \ldots$, we have

$$
\Psi_{n}(-m,-m, z)=\int z^{n} e^{z} \Gamma(m+1, z) d z
$$

Replacing $\Gamma(m+1, z)$ by its expansion as a truncated exponential and integrating termwise, we obtain

$$
\Psi_{n}(-m,-m, z)=m ! \sum_{k=0}^{m} \frac{1}{k !} \frac{z^{n+k+1}}{(n+k+1)}
$$

For $a=b=+m$, we have a two-step reduction procedure. First, from eq (30a) with $a=m$ and $b=m+1$ we have

$$
\Psi_{n}(m+1, m+1, z)=\frac{1}{m} \int z^{n-m} d z-\frac{1}{m} \Psi_{n}(m, m, z)
$$

where we have used the integrated form of eq (34) to eliminate $\Psi_{n}(m, m+1, z)$. This reduction is continued until $\Psi_{n}(1,1, z)$ is generated. Second, noting that

$$
U(1,1, z)=e^{z} E_{1}(z)
$$


multiplying by $z^{n}$ and integrating by parts, we obtain the reduction formula

$$
\Psi_{n}(1,1, z)=z^{n} e^{z} E_{1}(z)+\frac{z^{n}}{n}-n \Psi_{n-1}(1,1, z) .
$$

Equation (52) is applied $n$ times until $\Psi_{0}(1,1, z)$ is generated which, by integration of eq (51), is obviously given by

$$
\Psi_{0}(1,1, z)=\ln z+e^{z} E_{1}(z) .
$$

This same result is obtained from eq (47) with $a$ chosen as $1-m$ and the replacement under the integral sign of $\Gamma(1-m, x)$ by $x^{1-m} E_{m}(x)$.

For $b>a$, if $a \leqslant 0$, eq (41) is repeatedly applied until a series of terms $\Psi_{n^{\prime}}\left(0, b^{\prime}, z\right)$ is obtained which is given by eq (33a). With $a>0$, specifically choosing $a=m+1$ and $b=m+m^{\prime}+2$ in eq (30a), we have the recursion relation

$\Psi_{n}\left(m+1, m+m^{\prime}+2, z\right)=(m+1) \Psi_{n}\left(m+2, m+m^{\prime}+2, z\right)+\Psi_{n}\left(m+1, m+m^{\prime}+1, z\right)$.

This recursion is repeated until the right-hand side contains only terms with $b=a+1$, given by the integral of eq (34). The general result is then simply

$$
\Psi_{n}\left(m+1, m+m^{\prime}+2, z\right)=\frac{1}{m !} \sum_{k=0}^{m^{\prime}}\left(\begin{array}{c}
m^{\prime} \\
k
\end{array}\right)(m+k) ! \int z^{n-k-m-1} d z
$$

Finally, the case $a>b$ is either treated by the reduction formula eq (41) for $a \leqslant 0$ until $\Psi_{n^{\prime}}(0$, $\left.b^{\prime}, z\right)$ of eq (33) is obtained, or for $a>0$, by repeated use of eq (31a) until $a=0$ or $a=b=+m$ (considered in eqs (50)-(53) above) is reached. Often one can utilize eq (35) to reduce the parameters to lower orders before starting the recursions. As a simple example of the case $a>b$, we present the result for $\Psi_{2}(4,2, z)$

$$
\Psi_{2}(4,2, z)=\Psi_{1}(3,0, z)=\frac{z^{2}}{12}\left[(1+z)-z(2+z) e^{z} E_{1}(z)\right] .
$$

\section{4. $M_{p}(0 ; a, b, z)$ and $U_{p}(0 ; a, b, z)$}

Consider first the integral $M_{p}(0 ; a, b, z)$. When the series for $M(a, b, z)$ is integrated termwise, we find that

$$
M_{p}(0 ; a, b, z)=\sum_{k=0}^{\infty} \frac{(a)_{k}}{(b)_{k}} \frac{1}{k !} \frac{\Gamma(p+k+1)}{\Gamma(p+k+2)} z^{p+k+1}=\frac{z^{p+1}}{p+1}{ }_{2} F_{2}(p+1, a ; p+2, b ; z),
$$

provided that $b \neq 0,-1,-2, \ldots$. , and $p \neq-1,-2, \ldots$ We note that for $p=-m$, with $m=1,2$, . . .,

$$
\begin{aligned}
M_{-m}(0 ; a, b, z)=\frac{(a)_{m}}{(b)_{m}} \frac{z}{m !}{ }_{3} F_{3}(a & +m, 1,1 ; b+m, 2,1+m ; z) \\
& -\sum_{k=0}^{m-2} \frac{(a)_{k}}{(b)_{k}} \frac{1}{k !} \frac{1}{(m-k-1)} z^{1+k-m}+\frac{(a)_{m-1}}{(b)_{m-1}} \frac{1}{(m-1) !} \ln z .
\end{aligned}
$$

From the definition for $U(a, b, z)$ in terms of $M(a, b, z)$, eq (29), we have

$$
\begin{aligned}
U_{p}(0 ; a, b, z)=\frac{\pi}{\sin \pi b}\left[\frac{1}{\Gamma(1+a-b) \Gamma(b)}\right. & M_{p}(0 ; a, b, z) \\
& \left.-\frac{1}{\Gamma(a) \Gamma(2-b)} M_{p+1-b}(0,1+a-b, 2-b, z)\right] .
\end{aligned}
$$


Contiguous relations for the generalized hypergeometric functions, ${ }_{r} F_{q}$, are given by Rainville [8]. From the special cases for $r=q=2$, we obtain

$$
\begin{aligned}
& a M_{p}(0 ; a+1, b, z)=z^{p+1} M(a, b, z)-(p+1-a) M_{p}(0 ; a, b, z), \\
& (b-1) M_{p}(0 ; a, b-1, z)=z^{p+1} M(a, b, z)-(p+2-b) M_{p}(0 ; a, b, z),
\end{aligned}
$$

and for the $U_{p}(0 ; a, b, z)$, from eqs (59), (60), and (61), we have

$$
\begin{gathered}
a(1+a-b) U_{p}(0 ; a+1, b, z)=z^{p+1} U(a, b, z)-(p+1-a) U_{p}(0 ; a, b, z), \\
(b-a-1) U_{p}(0 ; a, b-1, z)=z^{p+1} U(a, b, z)-(p+2-b) U_{p}(0 ; a, b, z) .
\end{gathered}
$$

Finally, by integrating eq (1) by parts, we have the recursion relations

$$
\begin{gathered}
a M_{p+1}(0 ; a+1, b+1, z)=b\left[z^{p+1} M(a, b, z)-(p+1) M_{p}(0 ; a, b, z)\right], \\
a U_{p+1}(0 ; a+1, b+1, z)=(p+1) U_{p}(0 ; a, b, z)-z^{p+1} U(a, b, z) .
\end{gathered}
$$

For $p=$ integer $n, n=0,1,2, . ., M_{n}(0 ; a, b, z) \equiv \Phi_{n}(a, b, z)$ and $U_{n}(0 ; a, b, z) \equiv \Psi_{n}(a, b, z)$, the cases discussed in sections 2 and 3 respectively. For $p \neq$ integer, four cases arise for which the integrals can be evaluated in terms of elementary functions or in terms of $M$ 's or $U$ 's respectively. The first case is the trivial one with $a=0$. We obviously have, by eqs (6) and (33)

$$
M_{p}(0 ; 0, b, z)=U_{p}(0 ; 0, b, z)=\frac{z^{p+1}}{p+1}
$$

The second case, $a=p+2+m, m=0,1,2, \ldots$, is reduced by repeated use of eqs (60) and (62) to the lowest order terms $a=p+2$, where

$$
\begin{gathered}
M_{p}(0 ; p+2, b, z)=\frac{z^{p+1}}{p+1} M(p+1, b, z), \\
U_{p}(0 ; p+2, b, z)=\frac{z^{p+1}}{(p+1)(p+2-b)} U(p+1, b, z),
\end{gathered}
$$

which are directly obtainable from eqs (60) and (62) by setting $a=p+1$. Thirdly, if $b=p+1-m$, then use of eqs (61) and (63) leads to the lowest order terms $b=p+1$, where

$$
\begin{aligned}
& M_{p}(0 ; a, p+1, z)=\frac{z^{p+1}}{p+1} M(a, p+2, z), \\
& U_{p}(0 ; a, p+1, z)=\left(\frac{z^{p+1}}{p+1-a}\right) U(a, p+2, z),
\end{aligned}
$$

which we find from eqs (61) and (63) by setting $b=p+2$. Lastly, for $b-a= \pm$ integer, use of eqs (60) and (61) reduces $M_{p}$ to the case $a=b$ for which

$$
M_{p}(0 ; a, a, z)=\frac{z^{p+1}}{p+1} M(p+1, p+2, z),
$$

whereas, if $b-a=1,2, \ldots$, repeated application of eqs (62) and (63) reduces $U_{p}$ to the case $b=a+1$ for which

$$
U_{p}(0 ; a, a+1, z)=\int z^{p-a} d z .
$$

We note that if none of the above conditions are satisfied, then use of eqs (60)-(65) will reduce the integrals to a series of similar integrals with $p, a, b$ in the interval $(0,1)$. Further analytical reductions are not available. 


\section{The General Integrals $M_{p}(\alpha ; a, b, z)$ and $U_{p}(\alpha ; a, b, z)$}

Let us consider the differential equation

$$
z \frac{d^{2} y}{d z^{2}}+(b-z) \frac{d y}{d z}-a y=f(z)
$$

For $f(z)=0$, we have the well-known confluent hypergeometric equation for which $M(a, b, z)$ and $U(a, b, z)$ are solutions. Babister [6] defines two nonhomogeneous confluent hypergeometric functions $\theta_{\sigma}(a, b ; z)$ and $\Lambda_{\rho, \sigma}(a, b ; z)$ which are particular solutions of eq (70) for (i) $f(z)=z^{\sigma-1}$ and (ii) $f(z)=\exp (z) z^{\sigma-1}$ respectively. He further discusses series expansions, contiguous relations, recurrence formulas, differential properties and several definite and indefinite integrals for $\theta$ and $\Lambda$.

We shall presently express $M_{p}$ and $U_{p}$ in terms of the functions investigated by Babister. Consider the following special cases of eq (70),

and

$$
z \frac{d^{2} y}{d z^{2}}+(b-z) \frac{d y}{d z}-a y=\exp (\rho z) z^{\sigma-1}
$$

$$
z \frac{d^{2} u}{d z^{2}}+(b-z) \frac{d u}{d z}-a u=0
$$

with particular solutions $y=\Lambda_{\rho, \sigma}(a, b ; z)$ and $u=M(a, b, z)$, respectively. Multiplying eq (70a) by $u e^{-z} z^{b-1}$ and eq (70b) by $y e^{-z} z^{b-1}$, and subtracting, we obtain

$$
\begin{aligned}
\frac{d}{d z}\left[z^{b} e^{-z}\left(M(a, b, z) \frac{d}{d z} \Lambda_{\rho, \sigma}(a, b ; z)-\Lambda_{\rho, \sigma}(a, b ; z) \frac{d}{d z} M(a, b, z)\right)\right] & =e^{(\rho-1) z} z^{b+\sigma-2} M(a, b, z) .
\end{aligned}
$$

Integrating both sides of eq (71) and setting $b+\sigma-2=p$ and $\rho=\alpha+1$, we have

$$
\begin{aligned}
M_{p}(\alpha ; a, b, z)=z^{b} e^{-z}\left[M(a, b, z) \frac{d}{d z} \Lambda_{\alpha+1, p-b+2}(a, b ; z)\right. & \\
& \left.-\Lambda_{\alpha+1, p-b+2}(a, b ; z) \frac{d}{d z} M(a, b, z)\right] .
\end{aligned}
$$

From the differential properties of $M(a, b, z)$, eq $(5)$, and $\Lambda_{\rho, \sigma}(a, b ; z)[6$, p. 135]

$$
\frac{d}{d z} \Lambda_{\rho, \sigma}(a, b ; z)=(\sigma-1) \Lambda_{\rho, \sigma-1}(a+1, b+1 ; z)+\rho \Lambda_{\rho, \sigma}(a+1, b+1 ; z),
$$

we obtain an alternative expression for $M_{p}(\alpha ; a, b, z)$ of eq (72)

$$
\begin{aligned}
M_{p}(\alpha ; a, b, z)=z^{b} e^{-z} & {\left[(\alpha+1) M(a, b, z) \Lambda_{\alpha+1, p-b+2}(a+1, b+1 ; z)\right.} \\
& +(p-b+1) M(a, b, z) \Lambda_{\alpha+1, p-b+2}(a+1, b+1 ; z) \\
& \left.-\frac{a}{b} M(a+1, b+1, z) \Lambda_{\alpha+1, p-b+2}(a, b ; z)\right]
\end{aligned}
$$

Contiguous relations for the $\Lambda$ are given by Babister [6, p. 135]. Two interesting special cases of eq (74) are obtainable, when $\alpha=0$ and when $\alpha=-1$ due to the relations $[6, p .134]$

$$
\begin{gathered}
\Lambda_{0, \sigma}(a, b ; z)=\theta_{\sigma}(a, b ; z), \\
\Lambda_{1, \sigma}(a, b ; z)=e^{-i \pi \sigma} e^{z} \theta_{\sigma}\left(b-a, b, e^{i \pi} z\right) .
\end{gathered}
$$


The first is obtained from eq (74) with $\alpha=0$ and eq (75b) and gives an expression for the $M_{p}(0 ; a$, $b, z)$ of section 4 in terms of $\theta$ 's and $M$ 's

$$
\begin{aligned}
M_{p}(0 ; a, b, z)=z^{b} e^{i \pi(b-p)}\left[M(a, b, z) \theta_{p-b+2}\left(b-a, b+1 ; e^{i \pi} z\right)\right. & \\
-(p-b+1) M(a, b, z) \theta_{p-b+1}\left(b-a, b+1 ; e^{i \pi} z\right) & \\
& \left.-\frac{a}{b} M(a+1, b+1, z) \theta_{p-b+2}\left(b-a, b, e^{i \pi} z\right)\right] .
\end{aligned}
$$

Furthermore, from eq (57) and from the following expression [6, p. 121]

$$
\theta_{\sigma}(a, b ; z)=\frac{z^{\sigma}}{\sigma(\sigma+b-1)}{ }_{2} F_{2}(1, \sigma+a ; \sigma+1, \sigma+b ; z),
$$

we obtain

$$
\begin{aligned}
& { }_{2} F_{2}(p+1, a ; p+2, b ; z)=M(a, b, z){ }_{2} F_{2}(1, p-a+1 ; p-b+2, p+2 ;-z) \\
& +\frac{p+1}{p+2} \frac{z}{(p-b+2)} M(a, b, z){ }_{2} F_{2}(1, p-a+2 ; p-b+3, p+3 ;-z) \\
& \quad-\frac{a}{b} \frac{z}{(p-b+2)} M(a+1, b+1, z){ }_{2} F_{2}(1, p-a+2 ; p-b+3, p+2 ;-z) .
\end{aligned}
$$

The second case of interest is obtained from eq (74) with $\alpha=-1$ and eq (75a), and is given by [6, p. 131]

$$
\begin{aligned}
M_{p}(-1 ; a, b, z)=z^{b} e^{-z}\left[(p-b+1) M(a, b, z) \theta_{p-b+1}\right. & (a+1, b+1 ; z) \\
& \left.-\frac{a}{b} M(a+1, b+1, z) \theta_{p-b+2}(a, b ; z)\right] .
\end{aligned}
$$

$M_{p}(-1 ; a, b, z)$ can be related to the $M_{p}(0 ; a, b, z)$ discussed above and in section 4 , if we use Kummer's relation $[4,5]$

$$
e^{-z} M(a, b, z)=M\left(b-a, b, e^{i \pi} z\right)
$$

thus

$$
M_{p}(-1 ; a, b, z)=e^{-i \pi(p+1)} M_{p}\left(0 ; b-a, b, e^{i \pi} z\right) .
$$

The condition $b=a$ in eq $(81)$ gives

$$
M_{p}(-1 ; a, a, z)=\frac{z^{p+1}}{p+1}
$$

corresponding to the first case considered in section 4 , eq (66). The integral of eq (80) in addition to eq (5b) gives

$$
M_{0}(-1 ; a, b, z)=\frac{b-1}{a+1-b} e^{-z} M(a, b-1, z) .
$$


Cases two and three of section 4, eqs (67a) and (68a), allow for the analytical integration of $M_{p}(-1$; $a, b, z)$ when $b=a+p+2+m$ and $b=p+1-m$ for $m=0,1,2, \ldots$. , and reduce respectively to the lowest order terms

$$
\begin{gathered}
M_{p}(-1 ; a, a+p+2, z)=\frac{z^{p+1}}{p+1} e^{-z} M(a+1, a+p+2, z), \\
M_{p}(-1 ; a, p+1, z)=\frac{z^{p+1}}{p+1} e^{-z} M(a+1, p+2, z) .
\end{gathered}
$$

From case four of section 4 we can obtain the integrals $M_{p}(-1 ; \pm n, b, z)$ for $n=0,1,2, \ldots \ldots$ Specifically, for $n$ positive we have

$$
\begin{aligned}
M_{p}(-1 ; n, b, z)=z^{p+1} e^{-z}\left[(-1)^{n}\right. & \frac{(b-n)_{n}}{(p-b+2)_{n}} \frac{1}{p+1} M(1, p+2, z) \\
& \left.+\sum_{k=0}^{n-1}(-1)^{k} \frac{(b-n)_{k}}{(p-b+n+1-k)_{k+1}} M(n-k, b, z)\right] .
\end{aligned}
$$

For $n$ negative, rather than using the reduction formula eq (60), it is simpler to integrate term by term the finite polynomial $M(-n, b, z)$.

The analysis proceeds in a straight forward manner for the $U_{p}(\alpha ; a, b, z)$ if we use the definition of $U(a, b, z)$ in terms of $M$ from eq (29). We then have

$$
\begin{aligned}
U_{p}(\alpha ; a, b, z)=\frac{\pi}{\sin \pi b}\left[\frac{1}{\Gamma(1+a-b) \Gamma(b)}\right. & M_{p}(\alpha ; a, b, z) \\
& \left.-\frac{1}{\Gamma(a) \Gamma(2-b)} M_{p-b+1}(\alpha ; a-b+1,2-b, z)\right] .
\end{aligned}
$$

We first note that for $b=a+1$

$$
U_{p}(\alpha ; a, a+1, z)=M_{p-a}(\alpha ; 0,1-a, z)=\int z^{p-a} e^{\alpha z} d z .
$$

We also obtain from eq (83) for $\alpha=-1$ and eqs (26) and (82e) the case $b=a$

$$
U_{p}(-1 ; a, a, z)=\frac{1}{p+1}\left[z^{p+1} \Gamma(1-a, z)+\gamma(p-a+2, z)\right] .
$$

Analogous to eq $(82 \mathrm{~b})$ we find that by repeated use of eq $(31), U_{n}\left(-1 ; a^{\prime}, b^{\prime}, z\right)$ is reduced to a series of terms $U_{0}(-1 ; a, b, z)$, given by

$$
U_{0}(-1 ; a, b, z)=-e^{-z} U(a, b-1, z) .
$$

Corresponding to the cases $b=a+p+2+m$ and $b=p+1-m$ we have

$$
\begin{gathered}
U_{p}(-1 ; a, a+p+2+m, z)=-z^{p+1} e^{-z} \sum_{k=0}^{m}(-1)^{k} \frac{m !}{(m-k) !} \quad U(a+1+k, a+p+2+m, z), \\
U_{p}(-1 ; a, p+1-m, z)=-z^{p+1} e^{-z} \sum_{k=0}^{m} \frac{m !}{(m-k) !} U(a+1+k, p+2-m+k, z) .
\end{gathered}
$$

We note that for $b= \pm n$, care must be taken in the use of eqs (59) and (83). In this instance, the limiting forms for these equations must be used. For a detailed discussion of this point, the reader is referred to Erdélyi et al. [9]. 


\section{Appendix: Reduction to the Bessel Function Case}

If $b=2 a$, then $M(a, b, z)$ and $U(a, b, z)$ are related to the modified Bessel functions $I$ and $K$

$$
\begin{gathered}
M\left(\nu+\frac{1}{2}, 2 \nu+1,2 z\right)=\Gamma(\nu+1) 2^{\nu} z^{-\nu} e^{z} I_{\nu}(z), \\
U\left(\nu+\frac{1}{2}, 2 \nu+1,2 z\right)=\pi^{-1 / 2}(2 z)^{-\nu} e^{z} K_{\nu}(z) .
\end{gathered}
$$

Specializi g the parameters in eq (57) to the case $b=2 a$ and substituting from eq (A1), we have the indefinite integral

$$
\int z^{\mu} e^{ \pm z} I_{\nu}(z) d z=\frac{z^{\mu+\nu+1}}{(\mu+\nu+1) \Gamma(\nu+1)} 2^{-\nu_{2}} F_{2}\left(\mu+\nu+1, \nu+\frac{1}{2} ; \mu+\nu+2,2 \nu+1 ; \pm 2 z\right),
$$

in agreement with Luke [1, p. 96]. Equations (67a) and (82c) give special cases of eq (A3) with $\mu=-3 / 2$ and $\nu=p+(3 / 2)$ whereas eqs (68a) and (82d) give special cases of eq (A3) with $\mu=\nu=p / 2$.

Equations (67b) and (84d) of the text give rise to

$$
\begin{aligned}
\int z^{-3 / 2} e^{z} K_{p+(3 / 2)}(z) d z & =-\sqrt{2 \pi} \frac{(2 z)^{p+1}}{(p+1)(p+2)} U(p+1,2 p+4,2 z) \\
& =\frac{z^{-1 / 2} e^{z}}{(p+1)(p+2)}\left[(p+2-z) K_{p+(3 / 2)}(z)-z K_{p+(5 / 2)}(z)\right], \\
\int z^{-3 / 2} e^{-z} K_{p+(3 / 2)}(z) d z & =-\sqrt{2 \pi}(2 z)^{p+1} e^{-2 z} U(p+3,2 p+4,2 z) \\
& =\frac{z^{-1 / 2} e^{-z}}{(p+1)(p+2)}\left[(p+2+z) K_{p+(3 / 2)}(z)-z K_{p+(5 / 2)}(z)\right] ;
\end{aligned}
$$

whereas eqs $(68 \mathrm{~b})$ and $(84 \mathrm{e})$ yield

$$
\begin{aligned}
\int z^{\nu} e^{z} K_{\nu}(z) d z & =\sqrt{\pi} \frac{(2 z)^{\nu+1}}{(2 \nu+1)} z^{\nu} U(\nu+1 / 2,2 \nu+2,2 z) \\
& =\frac{e^{z} z^{\nu+1}}{(2 \nu+1)}\left[K_{\nu}(z)+K_{\nu+1}(z)\right] \\
\int z^{\nu} e^{-z} K_{\nu}(z) d z & =-\sqrt{\pi}(2 z)^{\nu} z^{\nu+1} e^{-2 z} U(\nu+3 / 2,2 \nu+2,2 z) \\
& =\frac{e^{-z} z^{\nu+1}}{(2 \nu+1)}\left[K_{\nu}(z)-K_{\nu+1}(z)\right]
\end{aligned}
$$

in agreement with the Handbook of Mathematical Functions. ${ }^{3}$ We note further that eq (84b) gives

$$
\int e^{-z} z^{-\nu} K_{\nu}(z) d z=\frac{1}{(2 \nu-1)} e^{-z} z^{1-\nu}\left[K_{\nu \cdot 1}(z)-K_{\nu}(z)\right] \quad(\nu \neq 1 / 2) .
$$

From eqs (74) and (78) we obtain indefinite integrals of the product of powers of $z$, Bessel functions, and $\exp (\alpha z)$ expressed in terms of the $\Lambda$ 's and $\theta$ 's of section 5. Equation (79) leads to

${ }^{3}$ See eqs. 11.3.15) and (11.3.16) in [4, chap. 11]. Luke [1, p. 97] gives an equivalent form for (A6) and (A7) when $\nu=0$. 
an interesting identity involving ${ }_{2} F_{2}$ 's

$$
\begin{aligned}
\left(\frac{z}{2}\right){ }^{\nu} e^{z}[\Gamma(\nu+1)]^{-1}{ }_{2} F_{2}(\nu+1 / 2, \mu+\nu+1 ; 2 \nu+1, \mu+\nu+2 ;-2 z) \\
=I_{\nu}(z){ }_{2} F_{2}\left(1, \mu+\frac{3}{2} ; \mu-\nu+1, \mu+\nu+2 ; 2 z\right) \\
-\frac{z}{(\mu-\nu+1)}\left[I_{\nu}(z)+I_{\nu+1}(z)\right]{ }_{2} F_{2}\left(1, \mu+\frac{3}{2} ; \mu-\nu+2, \mu+\nu+2 ; 2 z\right) .
\end{aligned}
$$

Finally, from eq (82b), we obtain a decomposition formula for ${ }_{2} F_{2}(a, b ; c, d ; z)$ when $a=1, c=2$, and $d=2 b$. We then have ${ }^{4}$

$$
z_{2} F_{2}(1, a+1 ; 2,2 a+2 ; z)=\frac{2}{a}(a+1 / 2)[M(a, 2 a+1, z)-1],
$$

for $a \neq-k / 2, k=0,1,2, \ldots$, or in terms of Bessel functions

$2 z_{2} F_{2}(1, \nu+1 / 2 ; 2,2 \nu+1 ; 2 z)=\frac{1}{\left(\nu-\frac{1}{2}\right)}\left[(2 / z)^{\nu} z e^{z} \Gamma(\nu+1)\left\{I_{\nu-1}(z)-I_{\nu}(z)\right\}-2 \nu\right]$.

\section{References}

[1] Luke, Y. L., Integrals of Bessel Functions (McGraw-Hill Book Co., New York, 1962).

[2] Kim, H., and Parr, R. G., One-Center Perturbation Approach to Molecular Electronic Energies, J. Chem. Phys. 49, 3071 (1968).

[3] Pan, Y. C., and Hameka, H. F., Use of Green Functions in Atomic and Molecular Calculations, J. Chem. Phys. 49, 2009 (1968).

[4] Handbook of Mathematical Functions, edited by M. Abramowitz and I. A. Stegun, National Bureau of Standards, Applied Mathematics Series No. 55, U.S. Government Printing Office, Washington, D.C., June (1964). See Chapter 13, pages 503-536, on Confluent Hypergeometric Functions.

[5] Slater, L. J., Confluent Hypergeometric Functions (Cambridge University Press, London, 1960).

[6] Babister, A. W., Transcendental Functions (The Macmillan Co., New York, 1967).

[7] Erdélyi, A., Magnus, W., Oberhettinger, F., and Tricomi, F. G., Higher Transcendental Functions (McGraw-Hill Book Co., New York, 1953), Vol. 2. See Chapter 9 for an extensive discussion of the incomplete gamma functions.

[8] Rainville, E. D., Special Functions (The Macmillan Co., New York (1960), pp. 82-84.

[9] Erdélyi, A., Magnus, W., Oberhettinger, F., and Tricomi, F. G., Higher Transcendental Functions (McGraw-Hill Book Co., New York, 1953), Vol. 1. See Chapter 6, section 6.7.1 for a discussion of the logarithmic case.

(Paper 74B2-321)

\footnotetext{
${ }^{4}$ Equation (A10a) is a particular example of the more general result derivable from the series representation of ${ }_{p} F_{q}$.
}

$$
z_{p+1} F_{q+1}\left(1, a_{p}+1 ; 2, b_{q}+1 ; z\right)=\left[{ }_{p} F_{q}\left(a_{p} ; b_{q} ; z\right)-1\right]\left(\prod_{i=1}^{p} a_{i}\right) /\left(\prod_{i=1}^{q} b_{i}\right) .
$$

